\title{
Atomistic simulations of crack nucleation and intergranular fracture in bulk nanocrystalline nickel
}

\author{
Ajing $\mathrm{Cao}^{*}$ and Yueguang $\mathrm{Wei}^{\dagger}$ \\ State Key Laboratory of Nonlinear Mechanics (LNM), Institute of Mechanics, Chinese Academy of Sciences, Beijing 100080, \\ People's Republic of China
}

(Received 22 March 2007; revised manuscript received 30 April 2007; published 23 July 2007)

\begin{abstract}
We report large scale molecular dynamics simulations of dynamic cyclic uniaxial tensile deformation of pure, fully dense nanocrystalline $\mathrm{Ni}$, to reveal the crack initiation, and consequently intergranular fracture is the result of coalescence of nanovoids by breaking atomic bonds at grain boundaries and triple junctions. The results indicate that the brittle fracture behavior accounts for the transition from plastic deformation governed by dislocation to one that is grain-boundary dominant when the grain size reduces to the nanoscale. The grain-boundary mediated plasticity is also manifested by the new grain formation and growth induced by stress-assisted grain-boundary diffusion observed in this work. This work illustrates that grain-boundary decohesion is one of the fundamental deformation mechanisms in nanocrystalline $\mathrm{Ni}$.
\end{abstract}

DOI: 10.1103/PhysRevB.76.024113

PACS number(s): 62.20.Mk, 62.25. $+\mathrm{g}$

Nanocrystalline (nc) metals (grain sizes typically less than $100 \mathrm{~nm}$ ) generally exhibit extremely high strength but disappointingly low ductility compared with their coarse-grained counterpart. ${ }^{1,2}$ It was proposed that this is mainly attributed to the difficulty in preparing high-quality nc materials in such tiny grain scales without flaws such as impurities, porosities, and internal stress. ${ }^{3,4}$ Recently, reports of elevated tensile elongation without sacrificing tensile strength were found in nc copper. ${ }^{5-7}$ The presumption was made that there is a crossover of fracture mechanism from ductile to brittle fracture transition. ${ }^{8}$ Meanwhile, both molecular dynamics (MD) simulations ${ }^{9}$ and experimental observations ${ }^{10}$ have suggested that a crossover from dislocation dominant plasticity to grain-boundary (GB) related plasticity (e.g., GB sliding and/or grain rotation) would occur when the grain sizes reach a critical value.

Although fracture behavior of nc face-centered-cubic (fcc) metals is of scientific importance, the evolution of damage and final fracture is only beginning to be understood in experimental observations. The understanding of the atomiclevel fracture mechanisms and failure model of nc materials is still lacking. Previous MD simulations ${ }^{11}$ have focused on investigating the fracture property with a pre-existing artificial crack inside grains. However little work is addressed upon the issue of the initial crack formation mechanism and thereby fracture properties in fully dense nc metals within the MD framework. ${ }^{12-18}$

In this work, large scale MD simulations are carried out with an embedded-atom method (EAM) potential for $\mathrm{Ni}$, which is based on first-principles calculations. ${ }^{19}$ The simulated sample is generated using a Voronoi construction, ${ }^{20}$ which contains 18 grains with an average grain diameter of $12 \mathrm{~nm}$ as shown in Fig. 1(a). To explore three-dimensional (3D) bulk behavior, periodical boundary conditions (PBC) are applied in all directions of the simulation cell.

Before tensile loading, the sample is first relaxed in the isobaric-isothermal ensemble under both the pressure 0 bar and temperature $300 \mathrm{~K}$ for $20 \mathrm{ps}$. Two respective loading simulations are performed in this work. In the first continuous loading, after equilibration processes, simulation starts until strain of $12 \%$ under the constant strain condition. The Melchionna equation of motion for the NPT ensemble governs the dynamics of the system, essentially using a combination of a Nosé-Hoover thermostat and a Parinello-Rahman barostat. $^{21,22}$ A constant strain rate of $2 \times 10^{8} \mathrm{~s}^{-1}$ is adopted in the $X$ direction and both boundary conditions in $Y$ and $Z$ directions are stress free, strain is introduced by uncoupling the unit cell vector along the $X$ direction from the dynamics and extending it during the simulation according to the applied strain rate. This is the same method used in Ref. 9 and 23. During the second loading-unloading-reloading cycle process, the constant strain rate of $2 \times 10^{8} \mathrm{~s}^{-1}$ is used until the stretched stain of $7.2 \%$, and then the unloading process is performed by stress relaxation within the Parrinello-Rahman approach, which is performed at $300 \mathrm{~K}$ and 0 bar constant stress for $100 \mathrm{ps,}$, and the strain is recorded with the stress during relaxation. After the unloading deformation, constant strain rate of $2 \times 10^{8}$ is adopted again to perform the reloading process until strain of $13.35 \%$. Although such a high strain rate (i.e., $2 \times 10^{8} \mathrm{~s}^{-1}$ ) is several orders of magnitude higher than the case used in a typical experimental tensile test, it is accepted to reach large strain within nanoscale time. 9,24

To discern grain boundary structure and other defects, we assign colors to the atoms according to a local crystallinity classification visualized by common neighbor analysis, ${ }^{25}$ which permits the distinction between atoms in a local hexagonal-close-packed (hcp) environment and those in a fcc environment. Gray stands for fcc atoms, red for hep atoms, green for other 12-coordinated atoms, and blue for the non12-coordinated atoms. A single line of hcp atoms represents a twin boundary, two adjacent hep lines stand for an intrinsic stacking fault, and two hep lines with an fcc line stand for an extrinsic stacking fault between them. For black and white figures, light gray represents fcc atoms and black represents other atoms.

Figure 1(b) shows the uniaxial tensile stress-strain curves for the two round simulations. In the continuous loading simulation, the true stress at the initial deformation stage shows linear increase with increasing true strain and the 
(a)

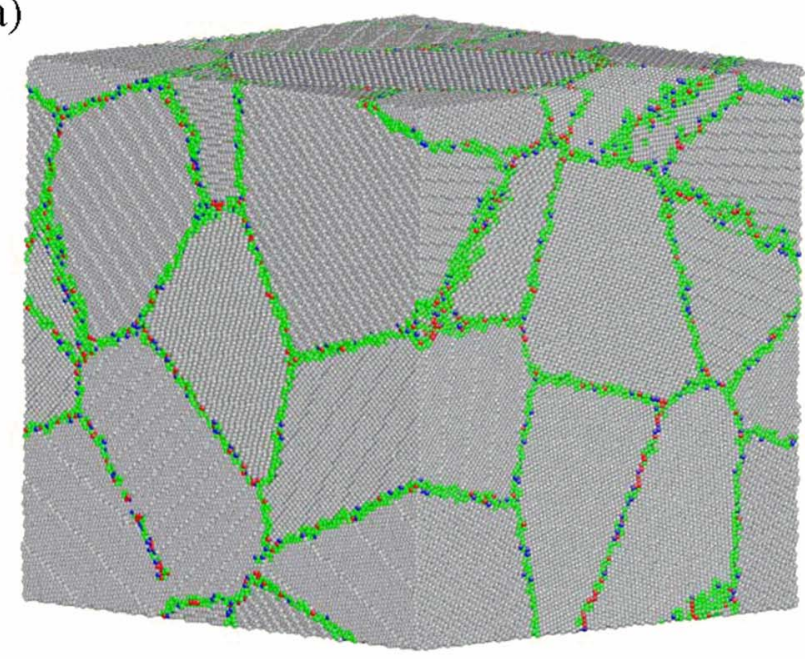

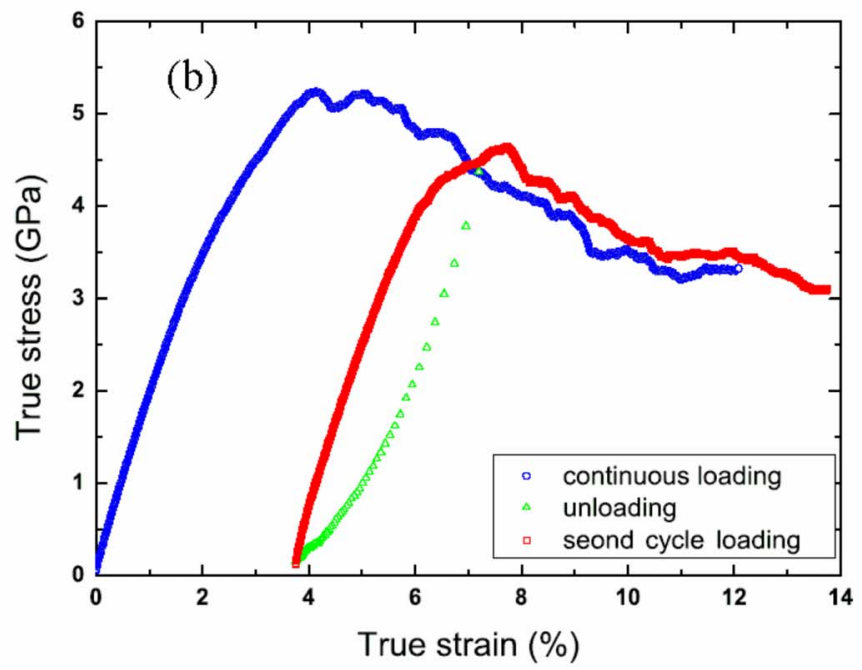

FIG. 1. (Color online) (a) Three-dimensional simulated sample configuration of the nc Ni corresponding to 18 grains with an average grain diameter of $12 \mathrm{~nm}$, containing 2.0 million atoms, which is relaxed at 0 bar pressure and $300 \mathrm{~K}$ temperatures. Atoms are colored according to common neighbor analysis. (b) Tensile stress-strain curve of nc Ni for a continuous loading simulation and a simulation containing complete loading-unloading-reloading cycle.

Young's modulus obtained from the stress-strain curve is $\sim 200 \mathrm{GPa}$. At the strain of about 3.2\%, the initial dislocations are emitted from the GBs, which indicates that plasticity has set in. After the stress reaches a maximum strength of $5.2 \mathrm{GPa}$ at $4 \%$ total strain, the curve shows a negative slope which is responsible for the formation of damage (nanovoids). The Young's modulus extracted from the second reloading curve shows no significant change with the first continuous loading, while the plastic deformation is a little influenced by the residual nanovoids defects created in the first loading process. The yielding strength of the second loading exhibits about $0.6 \mathrm{GPa}$ decreasing compared with the first continuous loading. The flow stress is little larger than the stress corresponding to the same strain in the first continuous loading. This is mainly because the residual stress in the sample is not relaxed to zero for a long enough time during unloading. The relative higher yielding strength of the simulations compared with the experiments may result in the high strain rate and the selected perfect dislocationfree microstructure. 9,24

After the unloading relaxation, the defects such as dislocations and stacking faults are stepped out of the sample and little dislocation network accumulation debris is left, which indicates that the reversible plastic deformation is quite different from their coarse-grained counterparts. The difference is also shown in the recent experiments, in which the reversible peak broadening was observed during uniaxial loading of electrodeposited $\mathrm{nc} \mathrm{Ni}$ with grain size of $30 \mathrm{~nm}$, by using in situ peak profile analysis. ${ }^{26}$

The most striking feature observed in the present work is the cracks nucleating in the strained nanograins and propagating with increasing strain. It is found that nanovoids and crack are both initiated in the two deformation simulation tests. Figures 2 and 3 illustrate crack path along the GBs in the first continuous and second cycle loading of nc Ni indi- cating an intergranular fracture. The crack path in the two loading deformation processes are almost the same. For simplicity of interpretation, we only concentrate on the second cyclic loading process.

Before mechanical loading, the sample is void free. While when the total strain reaches $7.75 \%$ during the second reloading, roughly corresponding to maximum stress, the nanovoids are detected at the GBs and triple junctions as (a)

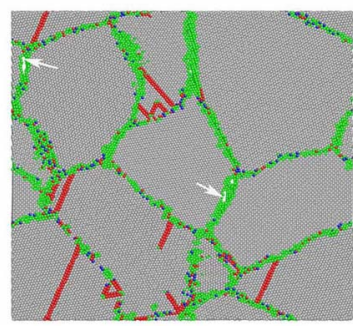

(c)

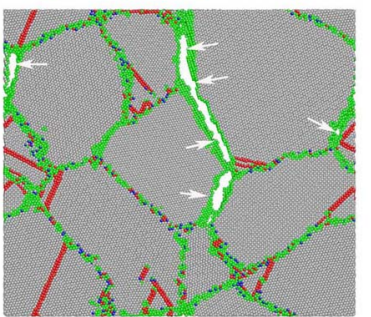

(b)

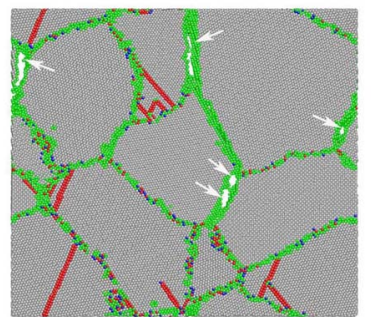

(d)

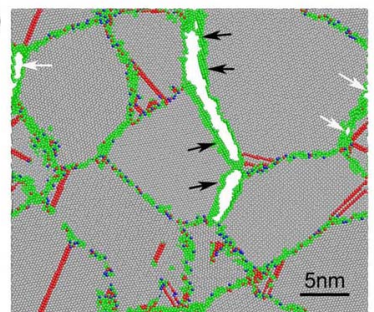

FIG. 2. (Color online) Crack nucleation in the first continuous loading simulation. Only a thin slab $(0.8 \mathrm{~nm})$ is shown. (a) At $\varepsilon$ $=7.2 \%$, nanovoids nucleated at different sites of GBs and triple junctions, which are indicated by the white arrow. (b) At $\varepsilon=8.8 \%$, (c) at $\varepsilon=10.4 \%$, nanovoids growth and coalescence by atomic bond decohesion. (d) At $\varepsilon=12 \%$, main crack (indicated by black arrow) formed by the coalescence of nanovoids at the GB region. Loading direction is in the horizontal axis. Atoms are colored according to common neighbor analysis. 
(a)

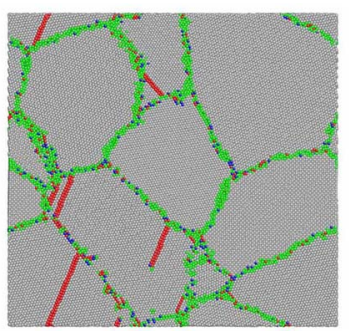

(c)

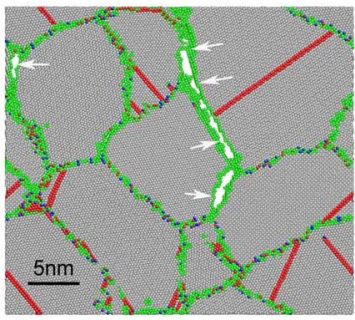

(b)

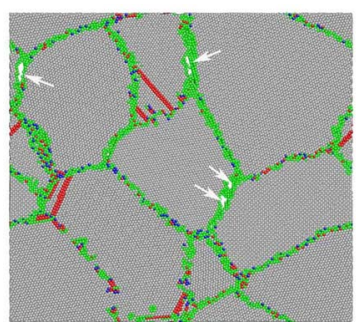

(d)

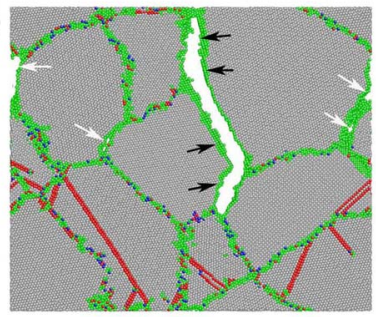

FIG. 3. (Color online) Crack path along GBs showing intergranular fracture in the second cycle loading simulation. Only a thin slab $(0.8 \mathrm{~nm})$ is shown. (a) At $\varepsilon=4.75 \%$, no nanovoids presented in the elastic deformation. (b) At $\varepsilon=7.75 \%$, nanovoids nucleation at different sites of GBs and triple junctions, which are indicated by the white arrow. (c) At $\varepsilon=9.35 \%$, nanovoids growth and coalescence by atomic bond decohesion. (d) At $\varepsilon=13.35 \%$, main crack (indicated by black arrow) formed by the coalescence of nanovoids at GB region and other nanovoids nucleate at different sites. Loading direction is in the horizontal axis. The dislocations, stacking faults, and deformation twins are also found in grains. Atoms are colored according to common neighbor analysis.

shown in Fig. 3(b). At this total strain, uniform deformation is exhausted. It is observed that nanovoids initiation occurs at many different sites, mainly at GB triple junctions. With further stretching, nanovoids coalesce and grow into the cracks [Figs. 3(c) and 3(d)]. Atomic bond breaking is the main reason for the coalescence of nanovoids. This may be caused by the incapability of providing sufficient local plastic deformation involving large number of grain boundary atoms. It should be noted that these dynamic atomic-scale processes would be nearly impossible to image with transmission electronic microscope (TEM).

To further quantitatively characterize the atomic level damage of the sample, the analysis is performed by evaluating the atomic level damage evolution. The damage on atom $i$ can be defined as the following formula according to firstorder coordination number of each atom:

$$
D_{i}=1-H\left(Z_{i}-Z_{\text {surf }}\right) \text {, }
$$

where $Z_{i}$ is the coordination numbers of the $i$ th atom, and $Z_{\text {surf }}$ is the coordination number of atoms in the surface environment [here it is 8 for face-centered-cubic (fcc) lattice]. $H\left(Z_{i}-Z_{\text {surf }}\right)$ is the standard step function, i.e., if $Z_{i}>Z_{\text {surf }}$, $H\left(Z_{i}-Z_{\text {surf }}\right)$ is 1 , otherwise $H\left(Z_{i}-Z_{\text {surf }}\right)$ is zero. By averaging the pointwise damage measure over a set of atoms in the sample, a cumulative measure of damage $D_{c}$ may be defined as

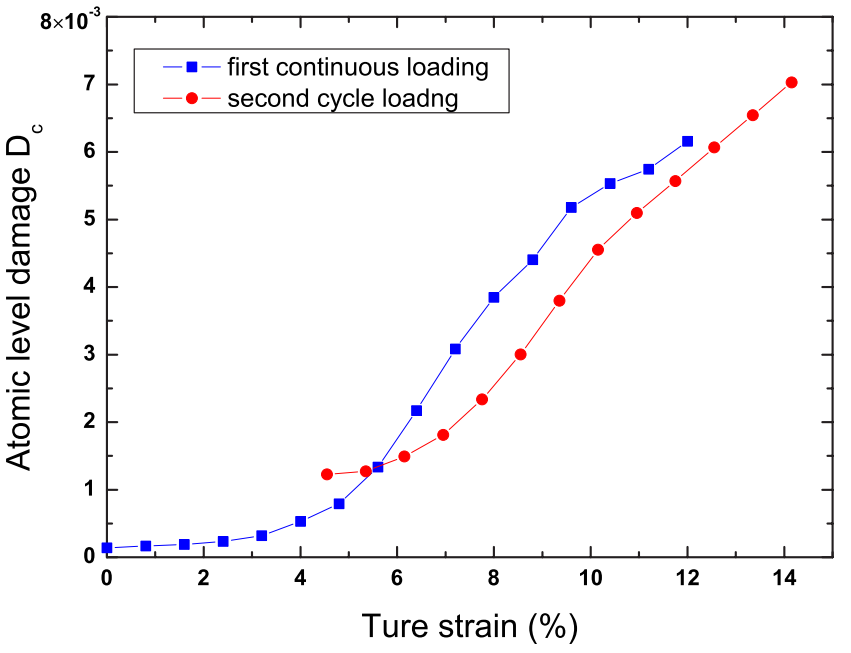

FIG. 4. (Color online) Atomic level damage evolution during the continuous loading and the second loading-unloading-reloading cycle.

$$
D_{c}=\frac{1}{N} \sum_{i=1}^{N} D_{i}=\frac{1}{N} \sum_{i=1}^{N}\left[1-H\left(Z_{i}-Z_{\text {surf }}\right)\right]
$$

here $N$ is the atoms in the whole sample.

The cumulative damage value $D_{c}$ demonstrates the nanovoids in the sample which are associated with newly formed free surfaces. Figure 4 shows the damage $\left(D_{c}\right)$ evolution during the two simulations. In the first loading test, during the initial deformation process (small strain), damage is not changed. The damage rises sharply at strain of $4.0 \%$ and then increases fast with the strain. At strain of $12 \%$ it is an order of magnitude higher than the initial one. In other words, the nanovoids accumulation is the reason for the final crack formation and macroscopic fracture. Clearly, beyond a strain of $4 \%$, fracture is the dominant deformation mechanism. The similar results could be found in the second cycle loading.

The spontaneous formation of intergranular cracks described in the present paper is in clear contrast to what has been reported earlier for the fcc Cu. Schiøtz et al. ${ }^{9}$ reports that MD simulations on copper with high tensile ductility do not show any formation of cracks within the investigated range of strains (up to $10 \%$ strain). During the deformation process, the dislocation density in the nc-Ni grains is very little where the high density of dislocations and stacking faults is found in $\mathrm{nc} \mathrm{Cu} .{ }^{9}$ Although the reason for this difference between fcc nc Ni and $\mathrm{Cu}$ is not well understood at present, the simulation results require more studies on the intrinsic different nature of materials even for the same fcc structure metals. It should be noted that previous atomistic simulations of the nc Ni (Ref. 24) with the constant high applied stress model is essentially high stress creeplike tests, and do not correspond to real constant strain rate tensile tests that were used in the experiments, which could not be used to investigate the fracture properties of nc materials (within $10 \%$ strain). Another factor we note is the different EAM potential used in our simulations and previous literature ${ }^{24}$ since the MD simulation results critically depend on the se- 
lected potential. The EAM potential used is based on $a b$ initio calculations and predicts a more precise generalized planar fault energy curve. ${ }^{19}$ The necking behavior is not seen in the simulations because of the small sample scale and the use of periodic boundary conditions. Unfortunately, we cannot provide a direct comparison with the deformation and fracture behavior of bulk Ni at larger grain sizes in our simulations, because they become too computationally expensive at these larger sizes.

Visualized snapshots and atomic-scale analysis of the simulations reveal that the strain is mainly localized in the GBs, whereas little dislocation and deformation twins are observed in the grains during the deformation process. This strongly indicates that the GB mediated plasticity is dominant, while the intragranular dislocation mediated plasticity contributes little to the whole plasticity. GB mediated plasticity can be accommodated in different ways, such as GB sliding, grain rotation, and also migration of the GB interface. It is suggested that cooperative GB sliding is accompanied by GB diffusion. ${ }^{27}$ Here in this work, we show that GB migration can play an active role in the plastic deformation. This can be demonstrated by the observation of the formation and growth of a new grain during straining. As shown in Fig. 5, in the initial deformation there are no grains formed in the area indicated by the white arrow [Fig. 5(a)]. After $4.0 \%$ strain deformation, a new small grain emerged from the triple junction near the white arrow [Fig. 5(b)]. At strain of $7.2 \%$, the new grain has grown into the approximate diameter of $5 \mathrm{~nm}$ [see Fig. 5(d)]. The grain growth rate will decrease with time, as a condition of new equilibrium is locally reached. Obviously, this new grain formation mechanism could be attributed to stress-assisted GB diffusion. It is well known that GB diffusion always takes place at elevated temperature. ${ }^{28}$ However, metal with nanometer-scale crystallites at room temperature should be capable of GB diffusion since the diffusion rate is increased dramatically as grain size decreases. The migration of GB observed indeed carries rotational plastic flow and this behavior would suppress the nucleation of nanocracks. ${ }^{27}$ This is evidenced by no cracks nucleated near the new formed grains (Fig. 5). A theoretical model has estimated that stress-induced GB migration can substantially contribute to plastic deformation of the nc materials and the mobility of the GB is dependent on the stress level. ${ }^{29}$ The present work could provide an atomic level evidence for the prediction. Another example of the nucleation of a new grain can be found from the previous work, which reported consequential dislocation emission mechanisms revealed by quasi-two-dimensional MD simulations. ${ }^{12}$

Many experiments have demonstrated that the dimple structure is often observed in the fracture surfaces in a nc metal with grain size of tens of nanometers, which is typical of polycrystalline metals during ductile fracture. ${ }^{8,30,31}$ How- (a)
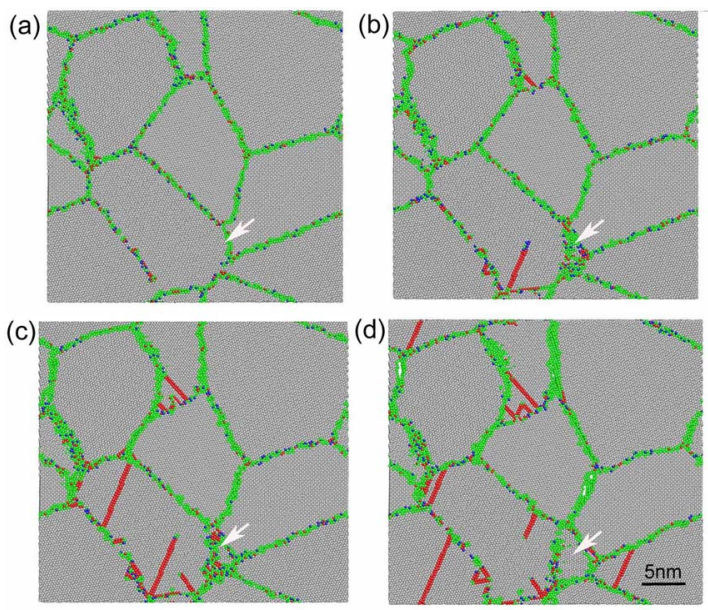

FIG. 5. (Color online) Consecutive snapshots showing formation of a new grain (near the white arrow) is accomplished with stress-assisted GB diffusion. Only a thin slab $(0.8 \mathrm{~nm})$ is shown. (a) Elastic deformation at strain of $\varepsilon=0.8 \%$, as evidence of no dislocations and stacking faults. (b) At strain $\varepsilon=4.0 \%$, a new grain is observed near the white arrow. (c) At strain of $\varepsilon=5.6 \%$, the new grain continuously grows with the size of about $2 \mathrm{~nm}$. (d) At strain of $\varepsilon=7.2 \%$, the new formed grain grows into approximately a diameter of $5 \mathrm{~nm}$. Atoms are colored according to the common neighbor analysis.

ever, experimental results concerning the fracture behavior of nc materials with a grain size below the critical value is very difficult and limited. ${ }^{8}$ This computation model sets up an image of GB dominant plasticity deformation mechanisms when the grain size is reduced to a critical value (for Ni 10-20 nm). A brittle fracture model with limited uniform deformation $(\sim 4 \%$ strain) predicted here in $\mathrm{nc} \mathrm{Ni}$ is in good agreement with experimental observations. ${ }^{8}$ This indicates that polycrystalline $\mathrm{Ni}$ behave as a brittle fracture with the grain size reduced to several nanometers even without artificial flaws. In view of this, the possible mechanical applications of $\mathrm{nc} \mathrm{Ni}$ would be limited when compared to other fcc nc metals. Furthermore, the atomic level interface de cohesion mechanism would provide fundamental information for a continuum plastic model with interface separation laws of nc metals. ${ }^{32,33}$

The authors are supported by the National Science Foundation of China through Grants Nos. 10432050, 10428207, 10672163, and Contract No. KJCX2-YW-M04 of the Chinese Academy of Sciences. The authors also acknowledge S. Plimpton from Sandia National Laboratory for sharing his MD code ParaDyn. ${ }^{34}$ The computations are partly supported by the Computing Facility for Computational Mechanics, Institute of Mechanics, Chinese Academy of Sciences.

\footnotetext{
*chaoajing@1nm.imech.ac.cn

†ywei@lnm.imech.ac.cn

${ }^{1}$ J. R. Weertman, D. Farkas, K. Hemker, H. Kung, M. Mayo, R. Mitra, and H. Van Swygenhoven, MRS Bull. 24, 44 (1999).
}

${ }^{2}$ Y. M. Wang, M. W. Chen, F. H. Zhou, and E. Ma, Nature (London) 419, 912 (2002).

${ }^{3}$ Y. M. Wang, E. Ma, and M. W. Chen, Appl. Phys. Lett. 80, 2395 (2002). 
${ }^{4}$ Y. T. Zhu and X. Z. Liao, Nat. Mater. 3, 351 (2004).

${ }^{5}$ K. M. Youssef, R. O. Scattergood, K. L. Murty, J. A. Horton, and C. C. Koch, Appl. Phys. Lett. 87, 091904 (2005).

${ }^{6}$ H. Q. Li and F. Ebrahimi, Appl. Phys. Lett. 84, 4307 (2004).

${ }^{7}$ H. Q. Li and F. Ebrahimi, Acta Mater. 54, 2877 (2006).

${ }^{8}$ H. Q. Li and F. Ebrahimi, Adv. Mater. (Weinheim, Ger.) 17, 1969 (2005).

${ }^{9}$ J. Schiøtz and K. W. Jacobsen, Science 301, 1357 (2003).

${ }^{10}$ Z. Shan, E. A. Stach, J. M. K. Wiezorek, J. A. Knapp, D. M. Follstaedt, and S. X. Mao, Science 305, 654 (2004).

${ }^{11}$ D. Farkas, H. Van Swygenhoven, and P. M. Derlet, Phys. Rev. B 66, 060101(R) (2002).

${ }^{12}$ V. Yamakov, D. Wolf, S. R. Phillpot, A. K. Mukherjee, and H. Gleiter, Nat. Mater. 1, 1 (2002).

${ }^{13}$ V. Yamakov, D. Wolf, S. R. Phillpot, A. K. Mukherjee, and H. Gleiter, Nat. Mater. 3, 43 (2004).

${ }^{14}$ H. Van Swygenhoven, Science 296, 66 (2002).

${ }^{15}$ H. Van Swygenhoven, P. M. Derlet, and A. G. Frøseth, Nat. Mater. 3, 399 (2004).

${ }^{16}$ H. Van Swygenhoven and P. M. Derlet, Phys. Rev. B 64, 224105 (2001).

${ }^{17}$ A. G. Frøseth, H. Van Swygenhoven, and P. M. Derlet, Acta Mater. 52, 2259 (2004).

${ }^{18}$ J. Schiøtz, Scr. Mater. 51, 837 (2004).

${ }^{19}$ Y. Mishin, D. Farkas, M. J. Mehl, and D. A. Papaconstantopoulos,
Phys. Rev. B 59, 3393 (1999).

${ }^{20}$ G. Z. Voronoi, J. Reine Angew. Math. 134, 199 (1908).

${ }^{21}$ M. Parrinello and A. Rahman, J. Appl. Phys. 52, 7182 (1981).

${ }^{22}$ S. Melchionna, G. Ciccotti, and B. L. Holian, Mol. Phys. 78, 533 (1993).

${ }^{23}$ A. J. Cao and Y. G. Wei, Appl. Phys. Lett. 89, 041919 (2006).

${ }^{24}$ A. Hasnouri, H. Van Swygenhoven, and P. M. Derlet, Science 300, 1550 (2003).

${ }^{25}$ J. D. Honeycutt and H. C. Andersen, J. Phys. Chem. 91, 4950 (1987).

${ }^{26}$ Z. Budrovic, H. Van Swygenhoven, P. M. Derlet, S. V. Petegem, and B. Schmitt, Science 304, 273 (2004).

${ }^{27}$ I. A. Ovid'ko and A. G. Sheinerman, Acta Mater. 53, 1347 (2005).

${ }^{28}$ R. L. Coble, J. Appl. Phys. 34, 1679 (1963).

${ }^{29}$ M. Yu. Gutkin and I. A. Ovid'ko, Appl. Phys. Lett. 87, 251916 (2005).

${ }^{30}$ R. C. Hugo, H. Kung, J. R. Weertman, R. Mitra, J. A. Knapp, and D. M. Follstaedt, Acta Mater. 51, 1937 (2003).

${ }^{31}$ K. S. Kumar, S. Suresh, M. F. Chisholm, J. A. Horton, and P. Wang, Acta Mater. 51, 387 (2003).

${ }^{32}$ V. Yamakov, E. Saether, D. R. Phillips, and E. H. Glaessgen, J. Mech. Phys. Solids 54, 1899 (2006).

${ }^{33}$ Y. J. Wei and L. Anand, J. Mech. Phys. Solids 52, 2587 (2004).

${ }^{34}$ S. J. Plimpton, J. Comput. Phys. 117, 1 (1995). 